\title{
Kinetic energy spectrum of horizontal motions in middle-atmosphere models
}

\author{
John N. Koshyk, ${ }^{1}$ Byron A. Boville, ${ }^{2}$ Kevin Hamilton, ${ }^{3}$ Elisa Manzini, ${ }^{4}$ and \\ Kiyotaka Shibata ${ }^{5}$
}

\begin{abstract}
Data from several middle-atmosphere general circulation models are used to calculate kinetic energy spectra as a function of total horizontal wavenumber $n$. The horizontal and vertical resolution between models varies but all have upper boundaries at heights $\gtrsim 80 \mathrm{~km}$. Tropospheric spectra show power-law behavior with slopes slightly shallower than -3 for wavenumbers $n \gtrsim 10$ (horizontal wavelengths $\lesssim 4000 \mathrm{~km}$ ) and are dominated by the rotational part of the flow. These spectra agree well with those calculated using data obtained from a global assimilation model and with the results of previous observational studies. Stratospheric spectra have larger amplitudes than tropospheric ones at planetary scales and smaller amplitudes at smaller scales. Mesospheric spectra are characterized by enhanced spectral amplitudes at all wavenumbers compared to the stratosphere and spectral slopes in the wavenumber range $n \gtrsim 10$ are generally shallower. Stratospheric and mesospheric spectra include approximately equal contributions from the rotational and divergent parts of the flow for $n \gtrsim 20$ in all models. These features appear to be independent of model resolution. The divergent part of the flow, presumably associated with explicitly resolved inertiogravity waves in the models, increases more rapidly with height above the lower stratosphere than the rotational part. The divergent part is fairly insensitive to season, whereas the rotational part changes considerably between January and July in the middle-atmosphere region. Spectral amplitudes and vertical growth rates of both parts vary widely between models for a given season. The horizontal diffusion schemes used by the models are compared in an attempt to explain some of these differences.
\end{abstract}

\section{Introduction}

The tropospheric kinetic energy (KE) spectrum as a function of horizontal wavenumber has been calculated from a variety of aircraft and assimilated data sources [Baer, 1972; Boer and Shepherd, 1983; Chen and WiinNeilsen, 1978; Koshyk and Boer, 1995; Nastrom et al., 1984]. On scales between about 4000 and $400 \mathrm{~km}$, the spectra show slopes of $\sim-3$, are dominated by the rotational part of the flow, and are associated with downscale enstrophy fluxes [Boer and Shepherd, 1983], all

\footnotetext{
${ }^{1}$ Department of Physics, University of Toronto, Toronto, Ontario, Canada.

${ }^{2}$ National Center for Atmospheric Research, Boulder, Colorado.

${ }^{3}$ Geophysical Fluid Dynamics Laboratory/NOAA, Princeton, New Jersey.

${ }^{4}$ Max-Planck-Institut für Meteorologie, Hamburg, Germany.

${ }^{5}$ Meteorological Research Institute, Tsukuba, Ibaraki, Japan.
}

Copyright 1999 by the American Geophysical Union.

Paper number 1999JD900814.

0148-0227/99/1999JD900814\$09.00 in close agreement with the theory of two-dimensional turbulence [Kraichnan, 1967] and, more generally, with the theory of geostrophic turbulence [Charney, 1971]. This spectral regime has been reproduced in general circulation model (GCM) simulations of the troposphere, subject to a reasonable choice of the horizontal subgrid-scale parameterization scheme [Koshyk and Boer, 1995; Koshyk et al., 1999; Laursen and Eliasen, 1989].

Calculations of the observed middle atmospheric $\mathrm{KE}$ spectrum as a function of horizontal wavenumber are limited to very large spatial scales because of relatively poorly resolved observations compared to the troposphere. Related work has focused on the power spectrum as a function of frequency at fixed points in the mesosphere using radar or lidar measurements [Balsley and Carter, 1982; Carter and Balsley, 1982; Gao et al., 1998; Vincent and Ball, 1981]. In these studies the KE has been found to vary as $\sim \omega^{-\alpha}$ where $\omega$ is the frequency and $1 \leq \alpha \leq 2$. Balsley and Carter [1982] found that $\alpha$ was very close to $5 / 3$, and used the Taylor hypothesis, which assumes $\omega \sim k U$ for a large-scale advection velocity $U$ and horizontal wavenumber $k$, to infer the existence of a $k^{-5 / 3}$ power law for KE in the mesosphere. They noted that this is the spectral slope in the upscale $\mathrm{KE}$ cascading inertial subrange of twodimensional turbulence theory [Kraichnan, 1967]. 
Table 1. Basic Features of the Models Used in This Study

\begin{tabular}{|c|c|c|c|c|c|}
\hline Model & $\begin{array}{l}\text { Horizontal } \\
\text { Resolution }\end{array}$ & $\begin{array}{c}\text { Number of } \\
\text { Vertical Levels }\end{array}$ & $\begin{array}{c}\text { Model Upper } \\
\text { Boundary (mbar) }\end{array}$ & $\begin{array}{c}\text { Model } \\
\text { Description }\end{array}$ & $\begin{array}{c}\text { Convective } \\
\text { Adjustment Scheme }\end{array}$ \\
\hline UKMO & $3.50^{\circ} \times 2.5^{\circ}$ & 21 & 0.32 & $\begin{array}{c}\text { Swinbank } \\
\text { and } O^{\prime} N e i l l[1994]\end{array}$ & $\begin{array}{c}\text { Gregory and } \\
\text { Rowntree [1990] }\end{array}$ \\
\hline CMAM & T32 & 50 & 0.00064 & $\begin{array}{c}\text { Beagley } \\
\text { et al. }[1997]\end{array}$ & $\begin{array}{c}\text { Zhang and } \\
\text { McFarlane [1995] }\end{array}$ \\
\hline MAECHAM4 & $\mathrm{T} 30$ & 39 & 0.01 & $\begin{array}{c}\text { Manzini } \\
\text { et al. }[1997]\end{array}$ & $\begin{array}{c}\text { Tiedtke }[1989] \\
\text { Nordeng }[1994]\end{array}$ \\
\hline MRI & $\mathrm{T} 42$ & 45 & 0.01 & $\begin{array}{l}\text { Shibata } \\
\text { et al. }[1999]\end{array}$ & $\begin{array}{c}\text { Moorth and } \\
\text { Suarez [1992] }\end{array}$ \\
\hline SKYHI (N30) & $3.6^{\circ} \times 3.0^{\circ}$ & 40 & 0.0096 & $\begin{array}{l}\text { Hamilton } \\
\text { et al. [1995] }\end{array}$ & $\begin{array}{l}\text { Holloway and } \\
\text { Manabe [1971] }\end{array}$ \\
\hline SKYHI (N90) & $1.2^{\circ} \times 1.0^{\circ}$ & 40 & 0.0096 & $\begin{array}{c}\text { Hamilton } \\
\text { et al. }[1995]\end{array}$ & $\begin{array}{l}\text { Holloway and } \\
\text { Manabe [1971] }\end{array}$ \\
\hline $\begin{array}{l}\text { MACCM2 } \\
\text { MACCM2 }\end{array}$ & $\begin{array}{l}\text { T42 } \\
\text { T106 }\end{array}$ & $\begin{array}{l}44 \\
44\end{array}$ & $\begin{array}{l}0.025 \\
0.025\end{array}$ & $\begin{array}{l}\text { Boville }[1995] \\
\text { Boville }[1995]\end{array}$ & $\begin{array}{l}\text { Hack [1994] } \\
\text { Hack [1994] }\end{array}$ \\
\hline
\end{tabular}

The U.K. Meteorological Office (UKMO) model is a troposphere-stratosphere data assimilation model, and the others are middle-atmosphere general circulation models. The notation "TN" for the horizontal resolution of spectral models refers to triangular truncation at total spherical harmonic wavenumber $N$. For the purposes of this study, data from the participating grid point models (UKMO and SKYHI) are transformed into spherical harmonic series with truncation T48 for UKMO, T50 for SKYHI (N30), and T150 for SKYHI (N90). The notation for SKYHI refers to the number of latitude rows between the pole and equator.

Hines [1960] has pointed out that much of the energy content of the middle atmosphere is provided by upward propagating inertiogravity waves. As a result, middle atmospheric KE spectra should contain a much stronger divergent component than is seen in the troposphere and should increase in amplitude with height (i.e., with decreasing density) above the lower stratosphere. Hamilton [1993] computed Fourier power spectra of the zonal wind from the Geophysical Fluid Dynamics Laboratory (GFDL) SKYHI middle-atmosphere GCM and found, using summer hemisphere midlatitude data, that spectral amplitudes do indeed increase with height between the lower stratosphere and the middle mesosphere. He ascribed the SKYHI spectral behavior to the presence of explicitly resolved stratospheric and mesospheric gravity waves, citing previous spectral analyses of SKYHI simulations [Hayashi et al., 1989; Miyahara et al., 1986].

In the present study, the KE spectrum and its rotational and divergent parts are computed for several different middle-atmosphere GCMs as a function of total spherical harmonic wavenumber $n$. Spectra are computed for the troposphere, as has been the focus of previous work [Boer and Shepherd, 1983; Koshyk and Boer, 1995; Koshyk et al., 1999], and also for the stratosphere and mesosphere. Assimilated data are used to validate the models in the troposphere and stratosphere, subject to the constraints placed on the assimilations by the resolution of the observing network and the effects of the assimilating models. In the mesosphere it is unclear how well the results presented here compare to reality, but an attempt to characterize them in terms of available theories is made.

\section{Models and Data Analysis}

The middle-atmosphere GCMs are versions of the Canadian Middle Atmosphere Model (CMAM), the Max Planck Institute for Meteorology Middle Atmosphere Model (MAECHAM4), the Japan Meteorological Research Institute Model (MRI), the GFDL Model (SKY$\mathrm{HI}$ ), and the National Center for Atmospheric Research (NCAR) Middle Atmosphere Community Climate Model (MACCM2). Assimilated data from the U.K. Meteorological Office (UKMO) troposphere-stratosphere assimilation model are also analyzed. Some basic characteristics of the models and references to more detailed descriptions of each are given in Table 1.

All models used in the study, with the exception of SKYHI and UKMO, are spectral models for which each prognostic variable is expanded in a triangularly truncated series of spherical harmonics,

$$
\psi(\lambda, \phi, p, t)=\sum_{n=0}^{N} \sum_{m=-n}^{n} \psi_{n}^{m}(p, t) P_{n}^{m}(\cos \phi) e^{i m \lambda}
$$

where $\psi$ is a prognostic model variable, $\lambda$ is longitude, $\phi$ is latitude, $p$ is pressure, $t$ is time, $m$ is the zonal wavenumber, $n$ is the total wavenumber, $N$ is the truncation wavenumber, and $P_{n}^{m}$ is the Legendre polynomial of degree $n$. Some properties of the spherical harmonics, $Y_{n}^{m}=P_{n}^{m} e^{i m \lambda}$, are given by Boer [1983]. The spectral coefficients, $\psi_{n}^{m}(p, t)$ are calculated by inverting (1),

$$
\psi_{n}^{m}(p, t)=\frac{1}{2 \pi} \int_{0}^{2 \pi} \int_{-1}^{1} \psi(\lambda, \phi, p, t) Y_{n}^{m *}(\lambda, \phi) d \mu d \lambda,
$$


where $\mu=\sin \phi$ and the asterisk denotes a complex conjugate.

The globally averaged horizontal $\mathrm{KE}$ on a fixed pressure level can be calculated from the horizontal velocity field:

$$
E(p, t)=\frac{1}{2}\langle\mathbf{v} \cdot \mathbf{v}\rangle
$$

where

$$
\mathbf{v}=(u, v)=\mathbf{k} \times \nabla \psi+\nabla \chi,
$$

and angle brackets denote an average over the sphere. The first and second terms on the right-hand side of (4) represent the rotational and divergent parts of the flow for stream function $\psi$ and velocity potential $\chi$. Noting that the vorticity $\zeta=\nabla^{2} \psi$ and the divergence $\delta=\nabla^{2} \chi$, the kinetic energy spectrum per unit mass $\left(E_{n}^{m}(p, t)\right)$ can be obtained directly from the spectral coefficients for vorticity $\zeta_{n}^{m}$ and divergence $\delta_{n}^{m}$ as follows:

$$
E_{n}^{m}(p, t)=\frac{1}{4} \frac{a^{2}}{[n(n+1)]}\left(\left|\zeta_{n}^{m}\right|^{2}+\left|\delta_{n}^{m}\right|^{2}\right),
$$

where $a$ is the Earth radius [Lambert, 1984]. Alternative expressions for $E_{n}^{m}$ have also been used in the literature [Baer, 1972; Tang and Orszag, 1978]. The differences between these and (5) for purely rotational flow are discussed by Boer and Shepherd [1983]. Beyond the largest decade of spatial wavenumbers $(n \gtrsim 10)$, all expressions yield very similar results. For the purposes of this study, (5) is particularly convenient because vorticity and divergence are prognostic variables for the spectral models considered, and the KE spectrum can be partitioned into its rotational and divergent parts in a straightforward manner.

Both SKYHI and UKMO are grid point models for which the prognostic variables are the horizontal winds $u$ and $v$. The KE spectrum is calculated by interpolating horizontal winds from the model grids to appropriate Gaussian grids, transforming each wind component to a series of spherical harmonics, and appropriately differentiating these expansions term by term to obtain series expansions for vorticity and divergence.

In the following sections, spectra are shown as a function of total wavenumber $n$ alone, by summing over the zonal wavenumber $m$; that is,

$$
E_{n}(p, t)=\frac{1}{4} \frac{a^{2}}{[n(n+1)]} \sum_{m=-n}^{n}\left(\left|\zeta_{n}^{m}\right|^{2}+\left|\delta_{n}^{m}\right|^{2}\right) .
$$

Monthly mean values for January or July only are considered, where the mean represents a sum of stationary and transient parts,

$$
\begin{aligned}
\overline{E_{n}(p, t)} & =E_{n}^{S}(p)+E_{n}^{T}(p) \\
& =\frac{1}{4} \frac{a^{2}}{[n(n+1)]} \sum_{m=-n}^{n}\left[\left(\left.\overline{\zeta_{n}^{m}}\right|^{2}+\left|\overline{\delta_{n}^{m}}\right|^{2}\right)\right. \\
& \left.\left.+\overline{\left(\left|\zeta_{n}^{m^{\prime}}\right|^{2}\right.}+\overline{\left|\delta_{n}^{m^{\prime}}\right|^{2}}\right)\right]
\end{aligned}
$$

the overbar denotes a monthly mean, and the prime denotes a deviation from the mean.

\section{Results and Discussion}

\subsection{Vertically Averaged Kinetic Energy Spectra}

Figure 1 shows vertically averaged spectra for the regions loosely referred to here as the troposphere (500100 mbar), stratosphere (100-1 mbar), and mesosphere (1-.01 mbar). The vertical averages are obtained by integrating (7) over the given layer with respect to pressure and then dividing by the pressure difference across the layer (i.e., the average is mass-weighted). The nondimensional value $n=1$ corresponds to a wavelength of approximately $40,000 \mathrm{~km}$. Solid lines in the top right-hand corners of Figure 1 and ensuing figure panels are for reference and have slopes of -3 and $-5 / 3$.

Models for which January data are available are represented in the top three panels of Figure 1; those for which July data are available are represented in the bottom three panels. Tropospheric and stratospheric spectra computed from UKMO assimilated data for January and July 1995 are indicated by thick, solid lines (note that the upper boundary of the assimilation model is at $\mathbf{0 . 3 2}$ mbar, so mesospheric spectra are not calculated from the UKMO data).

Tropospheric model spectra all have slopes $\sim-3$ in the wavenumber range $10 \lesssim n \lesssim 30$ for both January and July. This is in agreement with the UKMO spectra and with the results of previous observational studies [Baer, 1972; Chen and Wiin-Neilsen, 1978; Boer and Shepherd, 1983; Koshyk and Boer, 1995]. In January, good quantitative agreement between the observed, CMAM, and SKYHI spectra is seen, but the MAECHAM4 spectrum is consistently weaker over its resolved wavenumber range. The broad transition from a large-scale $\sim-3$ to a mesoscale $\sim-5 / 3$ slope in the SKYHI (N90) spectrum is consistent with the observational study of Nastrom et al. [1984] and is discussed in detail elsewhere [Koshyk et al., 1999]. In July all models show weaker tropospheric spectral amplitudes than the observations except at their largest (MACCM2) and smallest (MRI and MACCM2) resolved scales.

Stratospheric spectra have somewhat steeper powerlaw regimes compared to the troposphere for $10 \lesssim n \leqslant 20$ but are shallower at larger wavenumbers. This is particularly evident for the July cases. There is a wide variation between spectral amplitudes for different models in both January and July. For any given model, stratospheric spectral amplitudes are generally larger at planetary scales and weaker at smaller scales compared to tropospheric values.

Mesospheric spectra are characterized by enhanced amplitudes compared to the stratosphere at all scales and for all models, in both January and July. (Note that the MACCM2 T42 and T106 mesospheric spectra are further truncated to T32 and T60 because they 

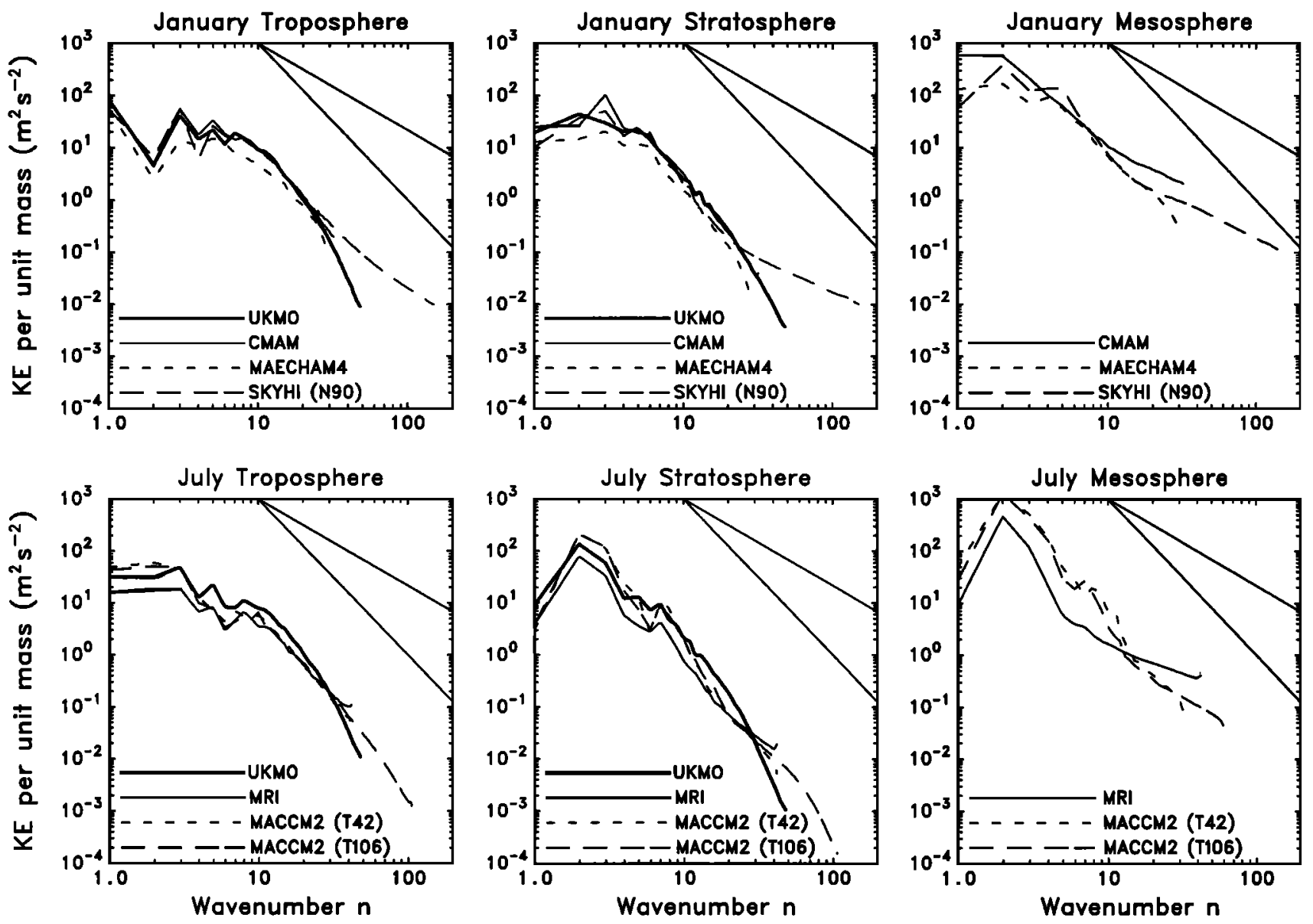

Figure 1. Monthly and vertically averaged kinetic energy (KE) per unit mass versus total horizontal wavenumber for six different middle-atmosphere general circulation models and for U.K. Meteorological Office (UKMO) assimilated data. The horizontal resolution of each model is given in Table 1. The vertical averages are taken over the following levels: troposphere, 500-100 mbar; stratosphere, 100-1 mbar; mesosphere, 1-.01 mbar. Straight lines in the top right-hand corner of each panel have slopes of -3 and $-5 / 3$. Models for which January/July data are available are represented in the top/bottom rows.

are affected by the excessive numerical dissipation discussed by Boville [1995].) Spectral slopes for wavenumbers $n \gtrsim 10$ are shallower taking values between -1 and -2. This agrees with the Fourier analysis performed by Hamilton [1993] using SKYHI data. The slopes for some models are, in fact, close to $\mathbf{- 5 / 3}$. Two-dimensional turbulence theory [Kraichnan, 1967] predicts the existence of a $-5 / 3$ upscale $\mathrm{KE}$ cascading inertial subrange, but it is unclear from the KE spectrum alone whether this theory is relevant here. The greatest discrepancies between model spectra for the three altitude ranges considered are found in the mesosphere.

Spectra calculated from assimilated data sources must be interpreted carefully, since the effective resolution of the atmospheric observing network is not precisely known. Thus it is difficult to determine where the UKMO spectra reflect the character of the assimilating model to a greater extent than they reflect the nature of the actual observations. The superexponential decrease in UKMO wave amplitudes for $n \gtrsim 30$ shown in
Figure 1 disagrees with all the models and is also inconsistent with analyses of horizontal spectra from aircraft observations [Nastrom et al., 1984].

Figure 1 indicates that the $\mathrm{KE}$ in the stratosphere generally takes greater values at planetary scales $(n \lesssim 5)$ and smaller values at larger wavenumbers compared to the troposphere in any given model. This is consistent with the Charney-Drazin criterion for quasi-geostrophic flow on a $\beta$ plane [Charney and Drazin, 1961]. For linear, conservative waves in an atmosphere with constant background wind and stratification, vertical propagation of stationary waves into the stratosphere is limited to the winter hemisphere and involves only planetary-scale eddies. Figure 2 shows tropospheric and stratospheric CMAM spectra for January similar to the CMAM spectra of Figure 1 but divided into contributions from the winter (northern) and summer (southern) hemispheres. The hemispheric spectra are computed using the method of Baer [1972] and Chen and Wiin-Neilsen [1978]. In the winter hemisphere, Figure 

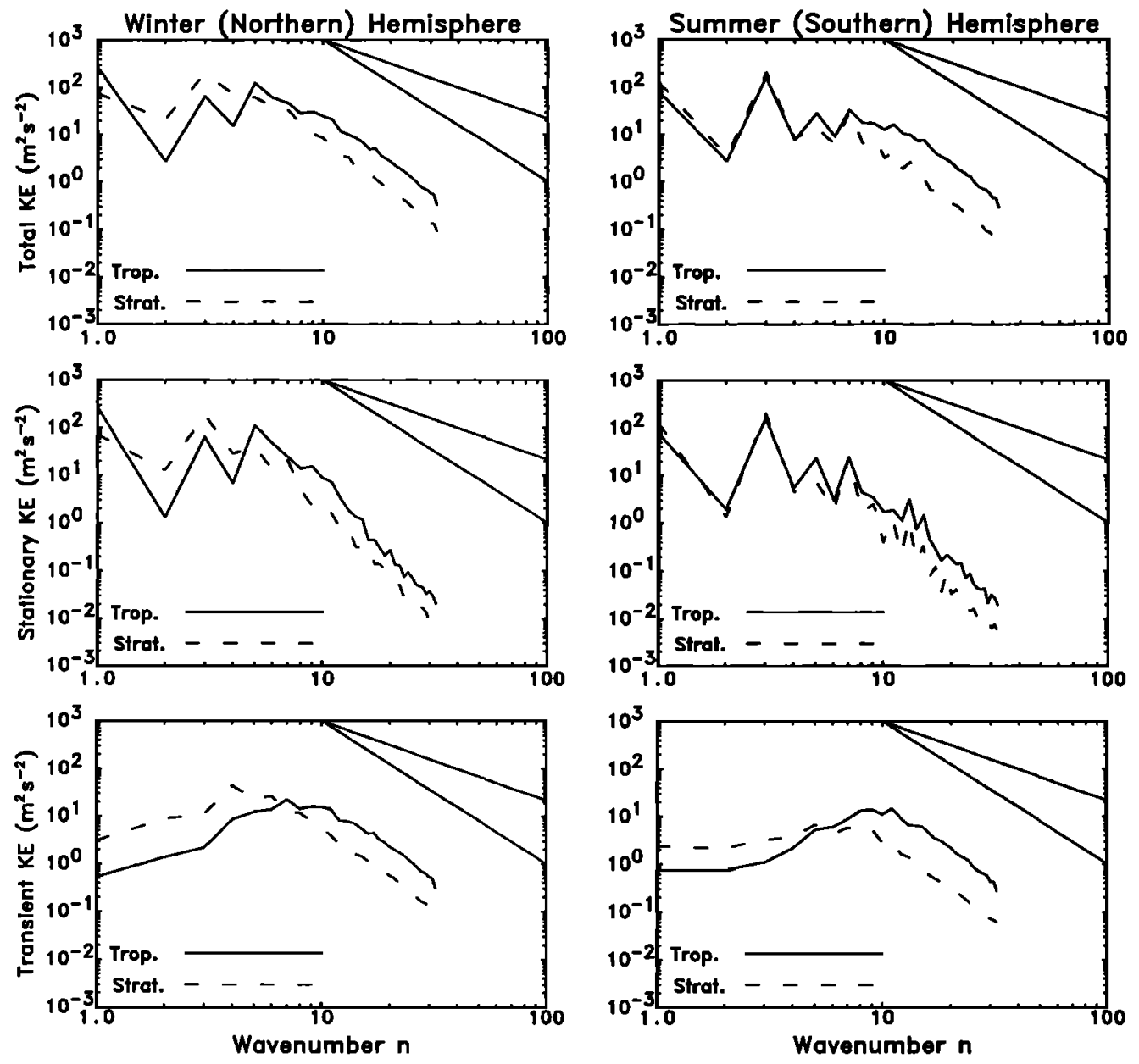

Figure 2. Hemispheric KE spectra for the CMAM model in January. Results for the winter (northern) hemisphere are shown in the left-hand column and for the summer (southern) hemisphere in the right-hand column. Total spectra are shown in the top row, their stationary component in the middle row, and their transient component in the bottom row. The curves represent vertically averaged quantities for the troposphere and stratosphere, as described in Figure 1.

2 shows a clear increase in total $\mathrm{KE}$ between the troposphere and the stratosphere for $2 \leq n \leq 4$ and a decrease in amplitudes for scales $n \geq 5$. (Note that when the zonal mean, or $m=0$ mode, is removed from the sum in (6), stratospheric spectral amplitudes exceed tropospheric amplitudes for all $n \leq 5$.) In the summer hemisphere, there is far less evidence of increased stratospheric amplitudes in the total KE at large scales. The middle and bottom panels of Figure 2 show the stationary and transient parts of the total KE spectrum in each hemisphere as defined in (7). The middle panels indicate that all but large-scale stationary waves in the winter hemisphere are trapped in the troposphere. This result is remarkably consistent with the CharneyDrazin criterion, even though the conditions for its validity are clearly not met in the model. The bottom panels show the transient component of $\mathrm{KE}$ in each hemisphere. The transient behavior of the spectra is similar in both hemispheres, with relatively larger stratospheric spectral amplitudes for $n \lesssim 5$ and relatively smaller stratospheric spectral amplitudes for $n>5$ compared to the troposphere. For traveling waves the vertical propagation characteristics depend on the horizontal phase speed, which is difficult to determine from the results presented here (although wave breaking in the lower stratosphere is consistent with the decreased spectral amplitudes at medium and small scales).

The seasonal dependence of the KE spectra in Figure 1 is unclear since different months correspond to a different set of models. Figure 3 shows KE spectra for the troposphere, stratosphere, and mesosphere for both January and July calculated from CMAM (left) and SKYHI (right) data. There are large differences between January and July at the planetary scales, since these scales are dominated by the stationary, topographically forced component of the flow which has a strong seasonal dependence. Similar results were obtained by Boer and Shepherd [1983]. Spectral amplitudes in the power-law regimes $(n \gtrsim 10)$ are nearly identical, with the largest differences for both models occurring in the stratosphere. The results from other models are similar. 

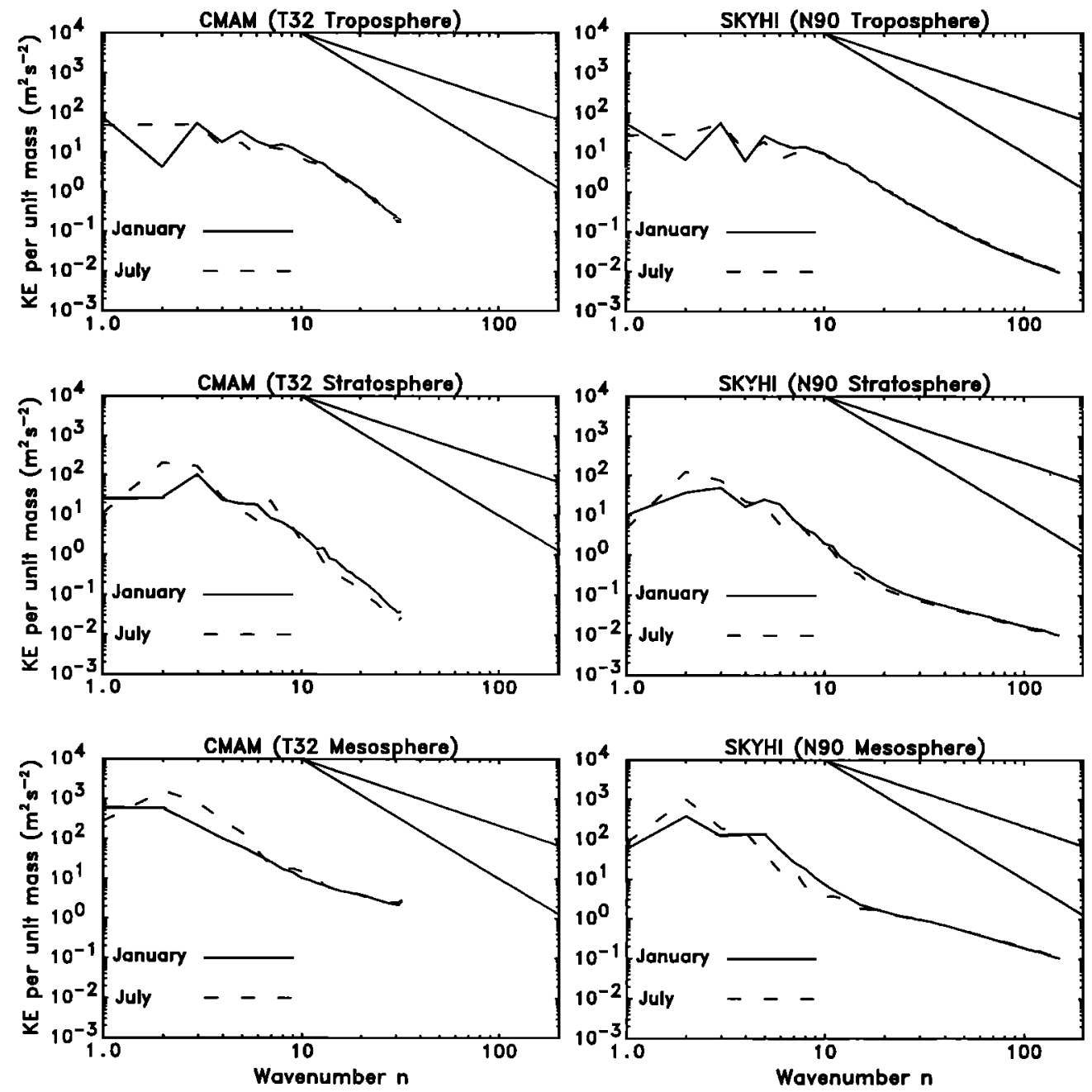

Figure 3. January and July monthly mean kinetic energy per unit mass versus total horizontal wavenumber for the CMAM model (left) and the SKYHI model (right). The curves represent vertically averaged values over the troposphere (top), stratosphere (middle), and mesosphere (bottom), as described in Figure 1.

\subsection{Rotational and Divergent Parts of the Kinetic Energy Spectrum}

As noted in the introduction, the amount of energy in the middle atmosphere at medium and high wavenumbers is determined mainly by upward propagating inertiogravity waves [Hines, 1960]. This differs from tropospheric dynamics at these scales, where the energy content is determined mainly by a downscale cascade of enstrophy (i.e. rotational wave modes) from a largescale baroclinic source [e.g., Boer and Shepherd, 1983]. The basic dynamical picture leads to the expectation that tropospheric KE spectra should be dominated by the rotational part of the flow, and the proportion of total $\mathrm{KE}$ in the form of gravity waves should increase with height, resulting in an enhanced divergent flow component in the middle atmosphere. The rotational part of the flow will generally also contain a gravity-wave or unbalanced component and should increase with height above the lower stratosphere as well.
Equation (6) can be partitioned into rotational and divergent parts by omitting divergence and vorticity spectral coefficients from the right-hand side. The resulting spectra in the troposphere, stratosphere, and mesosphere are shown in Figure 4 for January data and in Figure 5 for July data. There is a reasonably good quantitative agreement among the January models in all altitude ranges, with the largest differences in the mesosphere. In July, values of divergent $\mathrm{KE}$ predicted by the MACCM2 models are anomalously high over a broad wavenumber range, especially in the troposphere and stratosphere.

The top panels in Figures 4 and 5 show that the tropospheric $\mathrm{KE}$ is dominated by the rotational part of the flow at all scales, particularly the largest ones. One exception to this is the SKYHI curve for $n \gtrsim 100$, where rotational and divergent parts are comparable [Koshyk et al., 1999].

Stratospheric rotational KE spectra are marked by enhanced values at large scales and reduced values at 

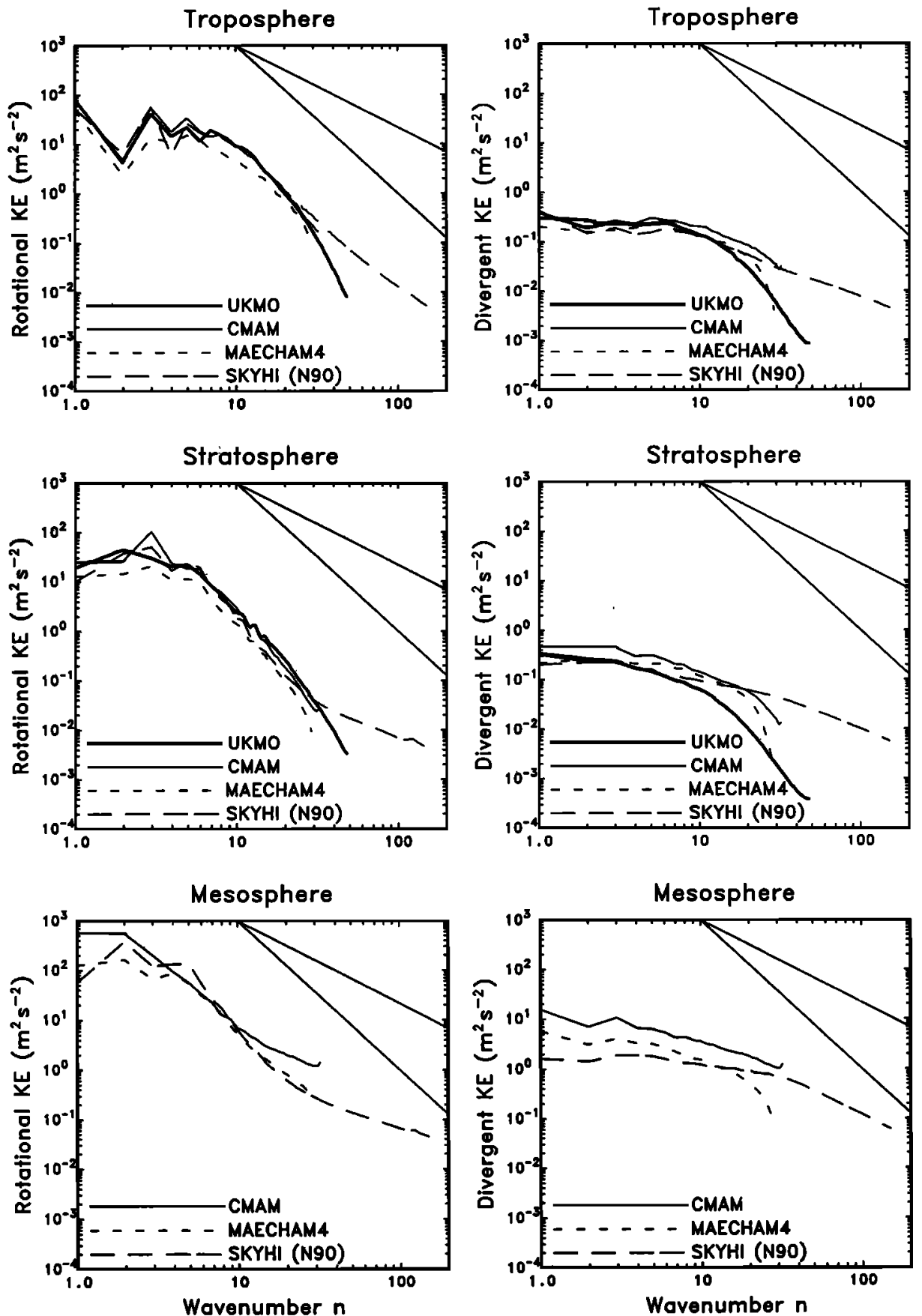

Figure 4. Rotational and divergent parts of the monthly mean kinetic energy per unit mass versus total horizontal wavenumber for January data from the UKMO assimilation, CMAM, MAECHAM4, and SKYHI (N90) models. The curves represent vertically averaged values over the troposphere (top), stratosphere (middle), and mesosphere (bottom), as described in Figure 1.

smaller scales compared to the troposphere. The smallscale filtering of rotational modes is consistent with the Charney-Drazin criterion mentioned in the previous subsection. The divergent part of the KE spectrum varies less than the rotational part between the troposphere and the stratosphere but becomes comparable to or greater than the rotational part for $n \gtrsim 20$.

The mesosphere contains increased levels of $\mathrm{KE}$ in both rotational and divergent components at all scales compared to the stratosphere. The divergent component of the flow is as large or larger than the rotational component for all wavenumbers $n \gtrsim 10$. Thus the shallow mesospheric slopes seen in Figure 1 are mainly the result of the more rapid increase of the divergent component compared to the rotational component with height. A wavenumber, $n=n_{*}$, can be defined such that the rotational and divergent components are comparable for $n \gtrsim n_{*}$. The value of $n_{*}$ is large in the lowermost 

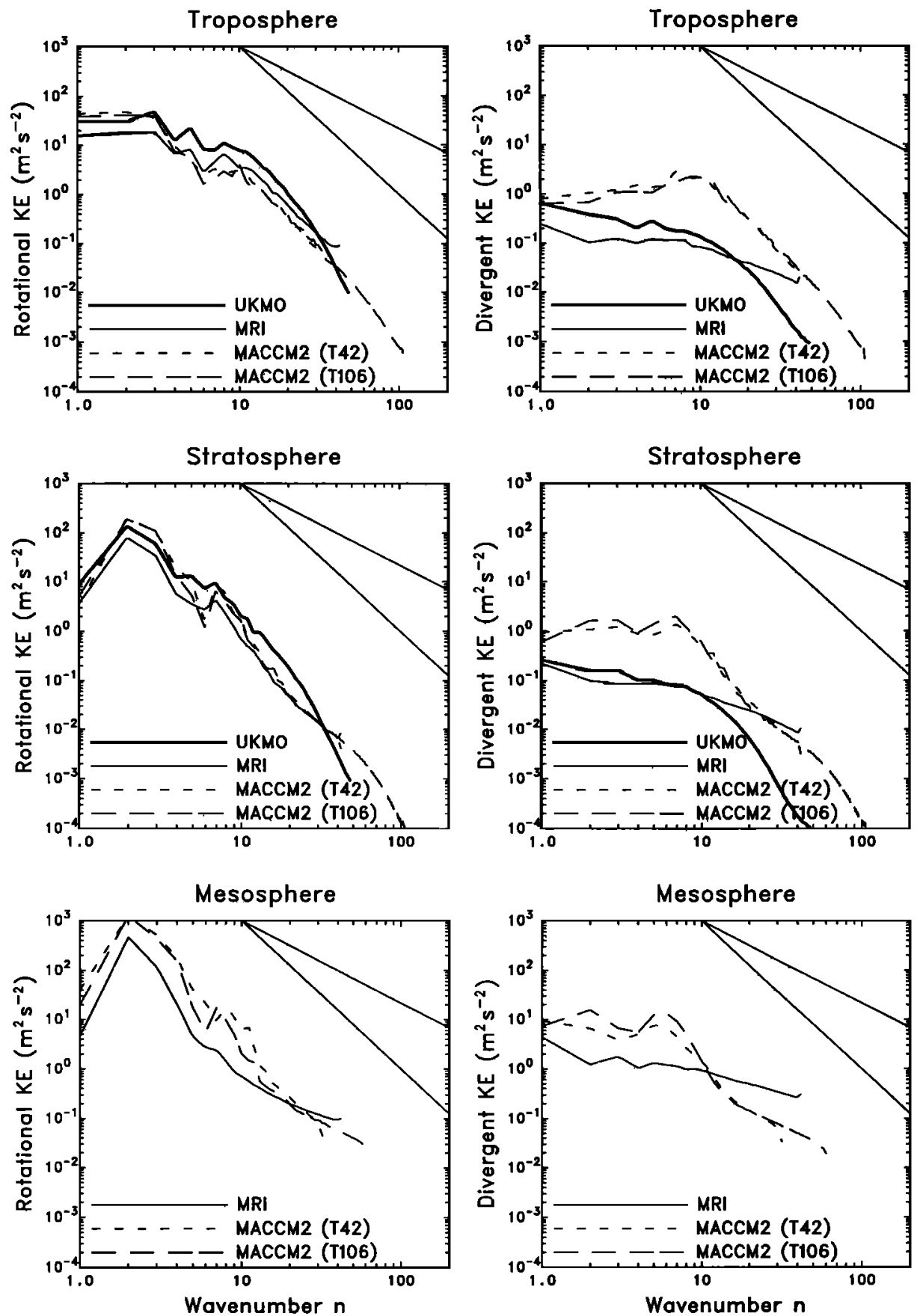

Figure 5. As Figure 4 but for July data from the UKMO assimilation, MRI, MACCM2 (T42), and MACCM2 (T106) models.

stratosphere and decreases monotonically with height to a value of $\sim 10$ in the mesosphere. Equivalently, if the atmospheric mesoscale is defined by the approximate equality $\delta \sim \zeta$, the spectral range $\left(n \gtrsim n_{*}\right)$ of the mesoscale regime becomes broader with height.

The increase in spectral amplitudes between the stratosphere and the mesosphere is consistent with the commonly held view that the middle atmospheric energy spectrum is determined largely by tropospherically generated upward propagating waves of all scales that amplify with height [Charney and Drazin, 1961; Hines,
1960]. It is beyond the scope of this study to actually characterize the waves contributing to the middle atmospheric spectra seen in all of the models. However, detailed space-time analyses of the SKYHI model [Hamilton and Mahlman, 1988; Hayashi et al., 1989] have shown that for sub-planetary scales, its spectrum is dominated by waves with relatively long vertical wavelengths that approximately satisfy the linear gravitywave dispersion relation [Andrews et al., 1987]. Since the large values of divergent $\mathrm{KE}$ in the stratosphere and mesosphere are consistent with a gravity-wave compo- 

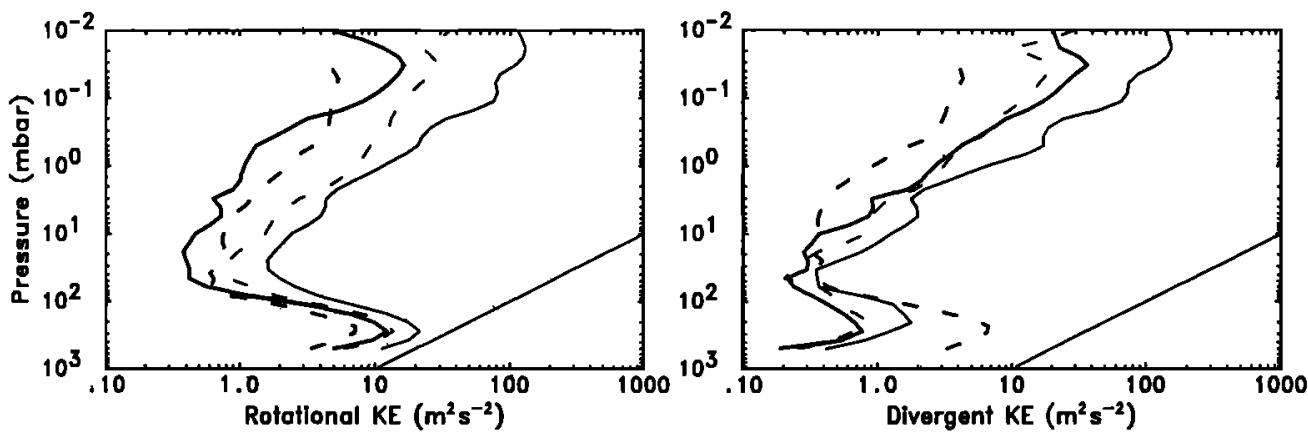

Figure 6. Rotational (left) and divergent (right) parts of the kinetic energy per unit mass as functions of pressure. Values at each pressure level are obtained by summing the spectrum over the wavenumber range $15 \leq n \leq 30$. The curves correspond to the models as follows: CMAM, January (thin solid line); MAECHAM4, January (thin dashed); MRI, July (thick solid line); MACCM2 T42, July (thick dashed). The straight line in the bottom right-hand corner of each panel has a slope of +1 .

nent in the flow, it is reasonable to assume that all of the models considered here contain a gravity-wave spectrum qualitatively similar to that of SKYHI.

According to conservative linear wave theory, assuming constant stratification and background wind, wave amplitudes in the middle atmosphere increase as $e^{z / 2 H}$, where $\mathrm{H}$ is a scale height [Andrews et al., 1987]. Thus in the simple linear case, a log-log plot of $\mathrm{KE}$ versus inverse pressure yields a straight line of slope +1 (i.e., $\mathrm{KE}$ $\sim e^{z / H}$ ). Figure 6 presents the vertical dependence of rotational (left-hand panel) and divergent (right-hand panel) KE components for the four lowest resolution models considered: CMAM in January (thin solid line), MAECHAM4 in January (thin dashed line), MRI in July (thick solid line), and MACCM2 (T42) in July (thick dashed line). The solid line in the bottom righthand corner of each panel is for reference and has a slope of +1 . At each pressure level, values in Figure 6 are computed by summing spectra over wavenumbers $15 \leq n \leq 30$, giving the contribution of scales in this range to the global average KE on that level. The lower summation bound is chosen to isolate the power-law regime in each model, minimizing the contributions of planetary waves that break in the stratosphere, and the upper bound corresponds to the truncation wavenumber of the lowest resolution model considered (MAECHAM4). The curves in Figure 6 corresponding to CMAM (T32), MRI (T42), and MACCM2 (T42) change very little when the upper bound is extended from $n=30$ to the truncation limit of each respective model.

Figure 6 shows that the models are similar in a qualitative sense, with local maxima near the tropopause and local minima in the lower stratosphere regions, for both rotational and divergent parts. The local maxima imply absorption or reflection of waves in the upper troposphere/lower stratosphere region and the decreased amplitudes above the local maxima are associated with wave breaking and saturation in the lower stratosphere. This agrees with observational evidence for topographically forced breaking gravity waves in the northern winter lower stratosphere [Barat, 1982; Sato and Woodman, 1982]. Above the lower stratosphere all models show an increase in amplitude with height right up to the middle mesosphere where gravity-wave breaking processes are expected to become particularly common. Part of the amplitude decrease in the mesosphere is associated with numerical "sponge" layers near the upper boundary of the models that strongly dissipate waves in order to prevent spurious reflections from the rigid lid.

Clear quantitative differences among the model results in Figure 6 exist. The rotational KE near the tropopause varies by almost a factor of 3 , while the divergent $\mathrm{KE}$ there varies by over an order of magnitude. Similar differences exist throughout the middleatmosphere region. Divergent KE grows at a faster rate than rotational KE for all models, but only the divergent parts of the CMAM and MRI spectra come close to the limiting +1 slope with height, with both increasing at a slightly slower rate. The energy density might be expected to grow more slowly than +1 because of gravity-wave breaking and saturation processes throughout the middle atmosphere, filtering of certain phase-speed ranges depending on the mean flow and parameterized dissipative processes in the models. Previous studies have shown that the Eliassen-Palm flux in GCM simulations decreases fairly rapidly with height throughout the middle atmosphere, an indication of wave breaking and saturation [Boville, 1986; Hamilton and Mahlman, 1988].

Precise reasons for the differences between models seen in Figure 6 are difficult to determine without a detailed spectral KE budget analysis of each. This would reveal the dominant processes that contribute to the $\mathrm{KE}$ at each wavenumber, including (1) horizontal transfers from other wavenumbers, (2) vertical fluxes of $\mathrm{KE}$ and potential energy into a vertical layer, (3) conversion from potential to kinetic energy, and (4) parameterized dissipative processes in the models (e.g., vertical and 

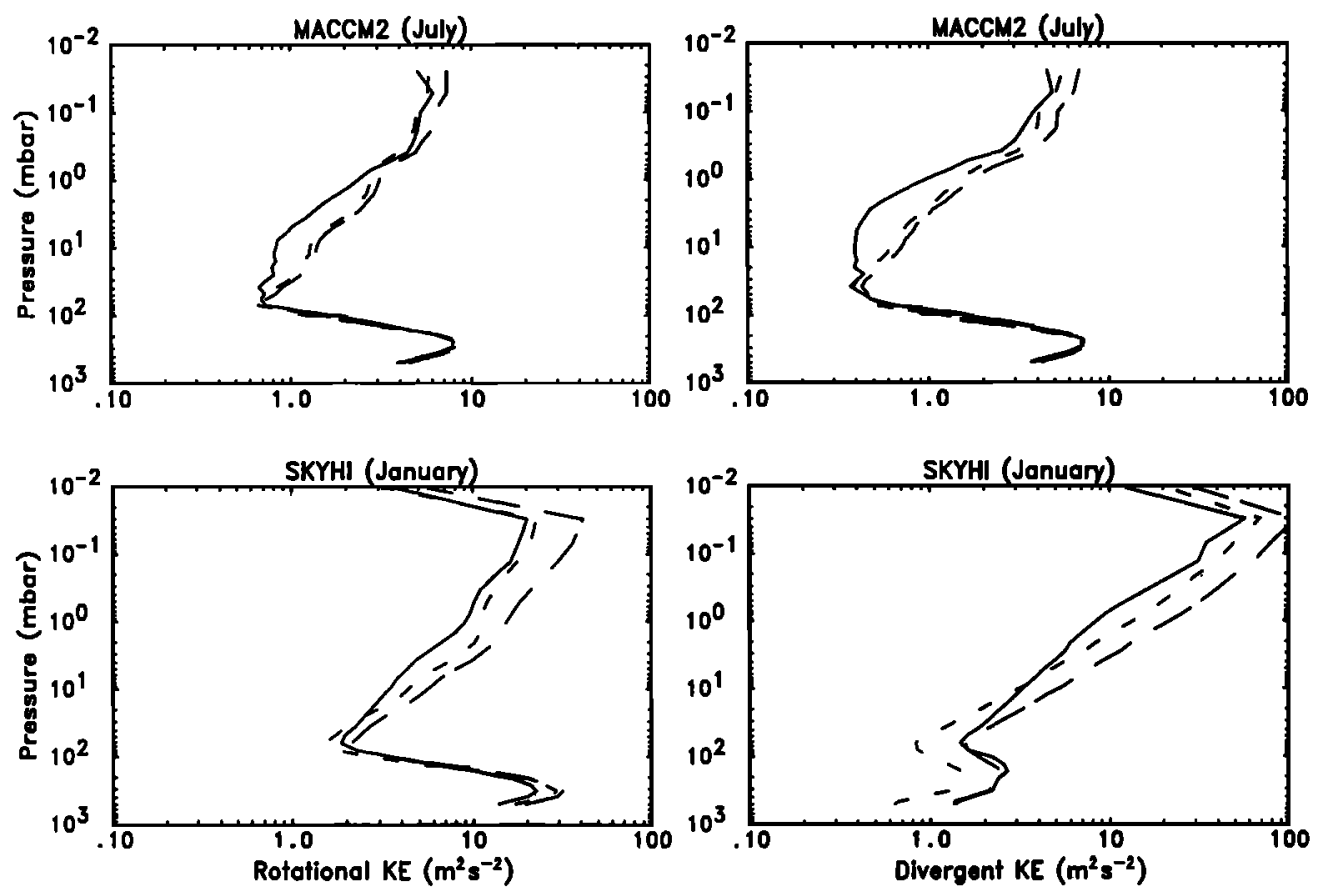

Figure 7. As Figure 6 but for MACCM2, July (top) and SKYHI, January (bottom). Values at each pressure level in the top panels are obtained by summing the MACCM2 T42 model from $n=15-42$ (solid line), the T106 model from $n=15-42$ (short dashed), and the T106 model from $n=15-106$ (long dashed). Values at each pressure level in the bottom panels are obtained by summing the SKYHI N30 model from $n=15-50$ (solid line), the N90 model from $n=15-50$ (short dashed), and the N90 model from $n=15-150$ (long dashed).

horizontal diffusion) [Lambert, 1984]. The studies of Hamilton and Mahlman [1988], Hayashi et al. [1989], and Miyahara et al. [1986] suggest that in the SKYHI middle atmosphere, vertical gravity-wave fluxes (i.e., as quantified by item 2) are important. Latent heating from the convective parameterization is a likely source of the resolved gravity wave field in the model simulations. The importance of this excitation was shown explictly for the SKYHI model [Manzini and Hamilton, 1993]. Differences in the convective parameterization schemes (Table 1) among models might account for some of the differences seen in Figure 6. It is also reasonable that differences in the parameterized vertical diffusion processes associated with wave breaking could be important. Finally, as discussed in the following section, all models use different scale-dependent horizontal diffusion parameterizations that increase monotonically toward the truncation wavenumber.

Figure 7 demonstrates the effects of model resolution on the fields calculated in Figure 6. The top panels show rotational and divergent KE for the MACCM2 T42 simulation with spectra summed from $n=15-42$ (solid), the MACCM2 T106 simulation with spectra summed from $n=15-42$ (short dashed), and the T106 simulation with spectra summed from $n=15-106$ (long dashed) at each pressure level. The high-resolution curves are qualitatively similar to their low-resolution counterparts. However, between the middle stratosphere and the lower mesosphere, there are fairly large differences between the T42 and the T106 curves in the $n=15-42$ range, especially in the divergent $\mathrm{KE}$. A possible reason for these differences is that the sub-gridscale parameterization schemes in the T42 simulation are not accurately representing the effects of unresolved scales (or, more accurately, of scales between $n=42$ and 106).

The bottom panels in Figure 7 display the same fields as the top panels but for SKYHI N30 summed from $n=15-50$ (solid), SKYHI N90 summed from $n=$ $15-50$ (short dashed), and SKYHI N90 summed from $n=15-150$ (long dashed) at each pressure level. As for MACCM2, noticeable differences between the highand the low-resolution curves are evident. The N30 model contains more divergent $\mathrm{KE}$ than the $\mathrm{N} 90$ model in the troposphere and lower stratosphere for the range $n=15-50$, suggesting that it is either underdissipated or too strongly forced (e.g., by gravity-wave sources) in that region. Differences between the $\mathrm{KE}$ in the $n=$ $15-50$ and $n=15-150$ ranges of the N90 simulations are greater than differences in the $n=15-42$ and $n=$ 15-106 ranges of the T106 simulation in the top panels, because the spectra in the SKYHI N90 simulation are not so steep as those in the MACCM2 T106 simulation at large wavenumbers.

Figure 8 compares the quantities shown in Figures 6 and 7 for January and July. The rotational and divergent KE spectra for CMAM summed from $n=15-32$ in both January and July at each level are shown in 

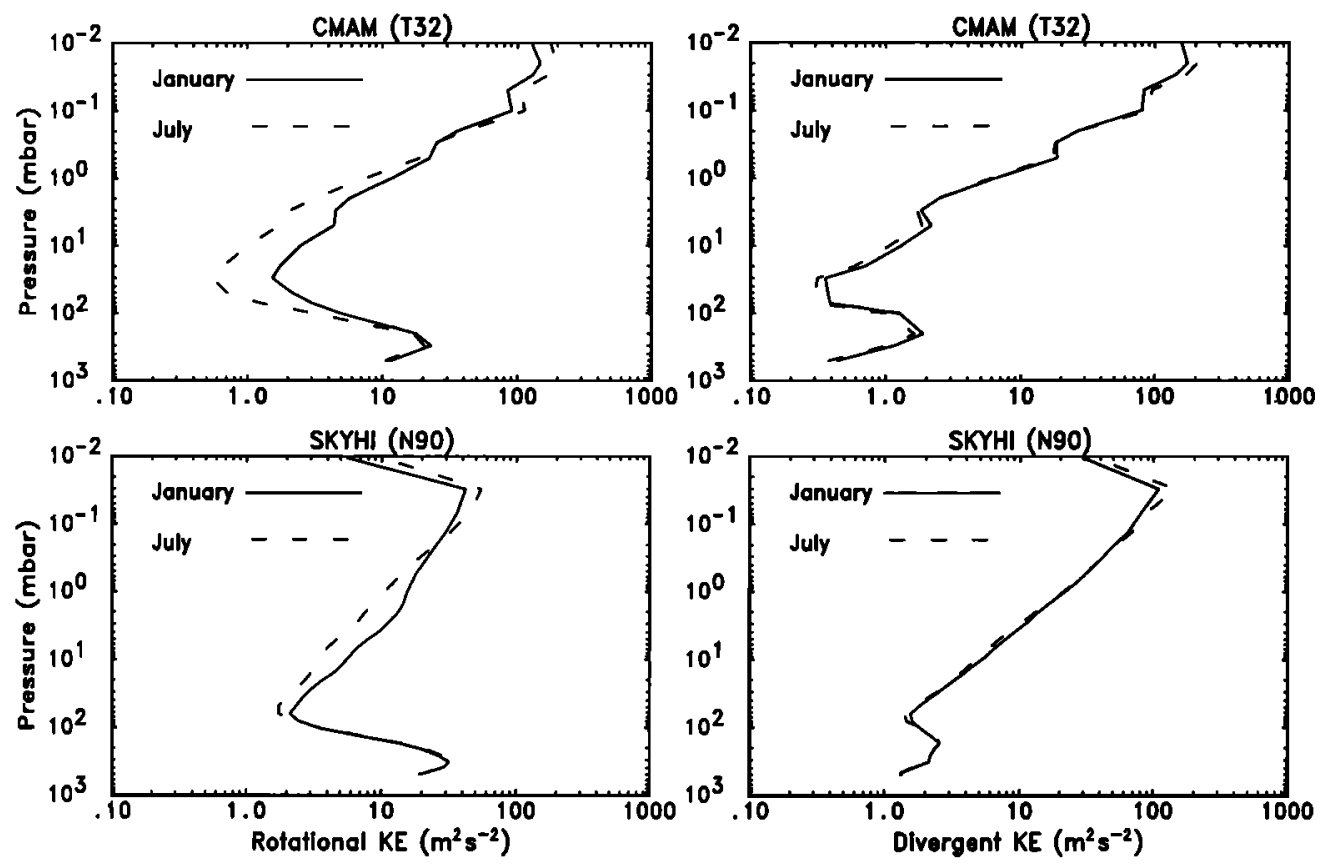

Figure 8. As Figure 6 but for CMAM (top), obtained by summing the spectrum from $n=15-32$ at each level, and SKYHI (bottom), obtained by summing the spectrum from $n=15-150$ at each level. Data for both January and July monthly means are shown in each panel.

the top panels. Corresponding curves for SKYHI N90 summed from $n=15-150$ at each level are shown in the bottom panels. The divergent KE is apparently insensitive to season in the wavenumber ranges considered. However, large differences in the rotational KE between January and July are evident in both models, especially in the stratospheric region. These seasonal differences account for some of the stratospheric variation in the left-hand panel of Figure 6, where the thin curves correspond to January and the thick curves to July for different models. Differences between January and July curves in the left-hand panels of Figure 8 are qualitatively similar, with generally lower stratospheric values and slightly higher mesospheric values in July compared to January. There is evidence that advection by planetary-scale flow is responsible for generating much of the small-scale structure often observed in the stratosphere [McIntyre and Palmer, 1983]. Since planetarywave activity is stronger in the Northern Hemisphere winter than in the Southern Hemisphere winter, it is possible that the enhanced spectral amplitudes seen in January are a result of more vigorous small-scale generation by the planetary-scale flow.

\subsection{Horizontal Diffusion Parameterizations}

The results of Figure 7 show that spectral amplitudes vary with horizontal resolution for a given model, particularly in the middle atmosphere. Reasons for the differences can plausibly be attributed to the failure of sub-grid-scale parameterization schemes in the lowerresolution models to accurately represent the effects of unresolved scales. Perhaps the most important such parameterization, especially for the medium and small scales considered in Figure 7, is the parameterization of horizontal sub-grid-scale dynamical processes. Since most middle-atmosphere GCMs are extensions of existing tropospheric models, the common practice is to extend the tropospheric horizontal sub-grid-scale parameterization into the middle atmosphere. With a few exceptions, tropospheric GCMs generally rely on diffusion or hyperdiffusion operators to represent the effects of horizontal subgrid scales by dissipating the horizontal downscale enstrophy cascade from the scales of baroclinic instability. In middle-atmosphere models, however, it is unclear whether spectral amplitudes at scales smaller than about $2000 \mathrm{~km}$ are primarily determined by horizontal downscale cascades. Rather, they may depend primarily on vertical wave fluxes from below [Hamilton and Mahlman, 1988; Hayashi et al., 1989].

Figure 9 shows the horizontal diffusion functions in spectral space for the relatively low-resolution models (left) and higher-resolution models (right). The MAECHAM4 model uses a $\nabla^{10}$ operator, and MRI and MACCM2 use $\nabla^{4}$ operators, each with differently specified coefficients. (Note that $\nabla^{2} Y_{n}^{m}=-\frac{n(n+1)}{a^{2}} Y_{n}^{m}$.) In addition, MACCM2 employs a strong horizontal spectral dissipation to control computational instability, mainly in the mesosphere region (not shown) [Boville, 1995]. The CMAM function is described by Boer et al. [1984], and is identically zero for $n=0-0.55 N$ (i.e., $n=0-18$ for T32 resolution) and a quadratic function for $n \geq .55 N$. It is based on the function proposed by Leith [1971] for a two-dimensional, homogeneous and isotropic turbulent fluid. The SKYHI model employs 

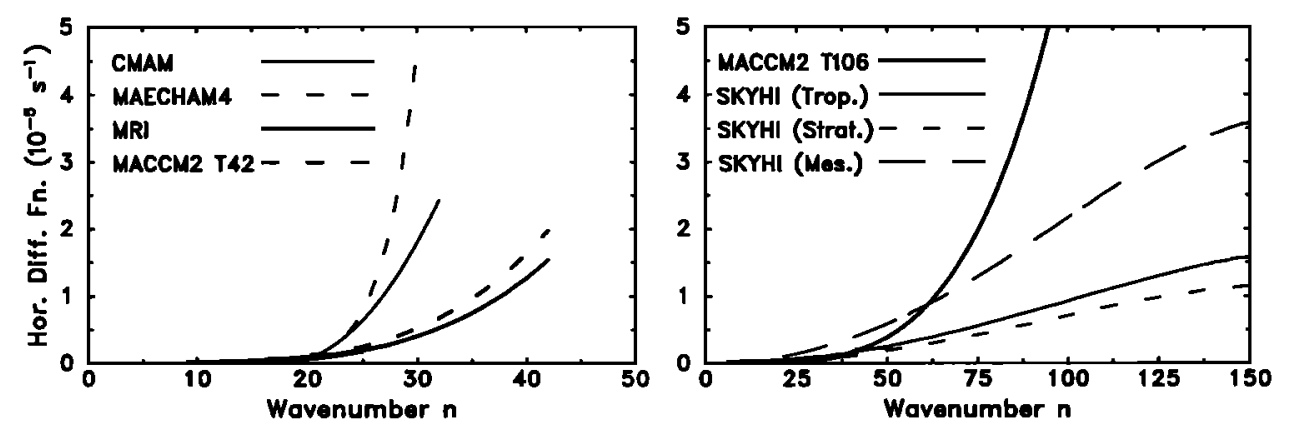

Figure 9. Spectral horizontal diffusion functions for the relatively low-resolution models considered in this study (left) and for the high-resolution models (right). Functional forms of the curves are given in the text. The SKYHI diffusion scheme is height-dependent and vertical means over the three representative model layers described in Figure 1 are shown. Note the difference in scale of the $x$ axis between the left and the right panels.

the Smagorinsky nonlinear horizontal eddy diffusivity parameterization [Andrews et al., 1983] for which an approximate spectral version is shown in Figure 9. The function is height-dependent because the diffusion coefficient depends on the local horizontal strain and deformation fields. The SKYHI curves are computed by calculating the horizontal diffusion of KE in spectral space from grid point values of the horizontal velocity and horizontal diffusion of momentum and then scaling this by the spectral KE at each level and wavenumber. Only vertical averages of the resulting quantity in the troposphere, stratosphere, and mesosphere are shown.

Figure 9 illustrates the difficulty in explaining the results seen in Figures 6 and 7 when examining only a single term in the KE budget. For example, although the CMAM horizontal diffusion is relatively strong compared to all the models except MAECHAM4, the CMAM curves (thin solid) in Figure 6 are consistently the most energetic for almost all wavenumbers, even after taking into account seasonal effects. Differences between the MRI (thick solid) and the MACCM2 T42 (thick dashed) curves in Figure 6 are also much greater than consideration of the horizontal diffusion functions alone would indicate. The right-hand panel shows that horizontal diffusion increases considerably between the stratosphere and the mesosphere in SKYHI, suggesting the possibility that the growth of spectral amplitude with height might be more limited by the horizontal diffusion in SKYHI than in the other models.

\section{Summary and Conclusions}

Data from several different middle-atmosphere general circulation models were used to compute kinetic energy spectra as a function of height and total spherical harmonic wavenumber $n$. The models agree with each other in a qualitative sense, showing the familiar $\sim-3$ slope for $n \gtrsim 10$ in the troposphere, enhanced values of stratospheric kinetic energy at planetary scales and diminished values at smaller scales compared to the troposphere, and enhanced spectral amplitudes at all scales in the mesosphere compared to the stratosphere. Spectral slopes in the range $n \gtrsim 10$ generally become shallower with height in all models.

Partitioning of the spectra into rotational and divergent parts showed that the divergent part grows more rapidly with height than the rotational part between the lower stratosphere and the mesosphere. For scales $n \gtrsim 20$, these components are comparable in magnitude above the lower stratosphere in each of the models. The divergent component appeared insensitive to season for the two months (January and July) considered, but the rotational component in the stratosphere clearly differed between these two months. Quantitatively, a wide variation in the energy content of the models at all scales was found throughout the upper troposphere and middle atmosphere region. This may be due to the differences in gravity-wave generation mechanisms (e.g., convective parameterization schemes) and propagation mechanisms (e.g., numerical advection and timestepping schemes) among the models.

Comparison of spectra from high- and low-resolution versions of the same model showed that sub-grid-scale parameterization schemes were not adequately representing the effects of unresolved scales in the middle atmosphere. In particular, it was assumed that the horizontal sub-grid-scale parameterization of unresolved processes (commonly referred to as horizontal diffusion) was largely responsible for the discrepancies, since it is expected to play a dominant role at medium and small scales. That the horizontal sub-grid-scale schemes appear to fail in the middle atmosphere is not surprising, since such schemes have been developed mainly on the basis of experience with tropospheric GCMs. The development of suitable horizontal subgrid-scale parameterization schemes for middle-atmosphere GCMs provides an extremely interesting area for future study.

In closing, it is illuminating to relate the $\mathrm{KE}$ spectra computed here to physical space fields. Figure 10 shows a snapshot of the SKYHI (N90) zonal wind field $u$ in July at three different model levels between the lower stratosphere and the lower mesosphere. Consis- 

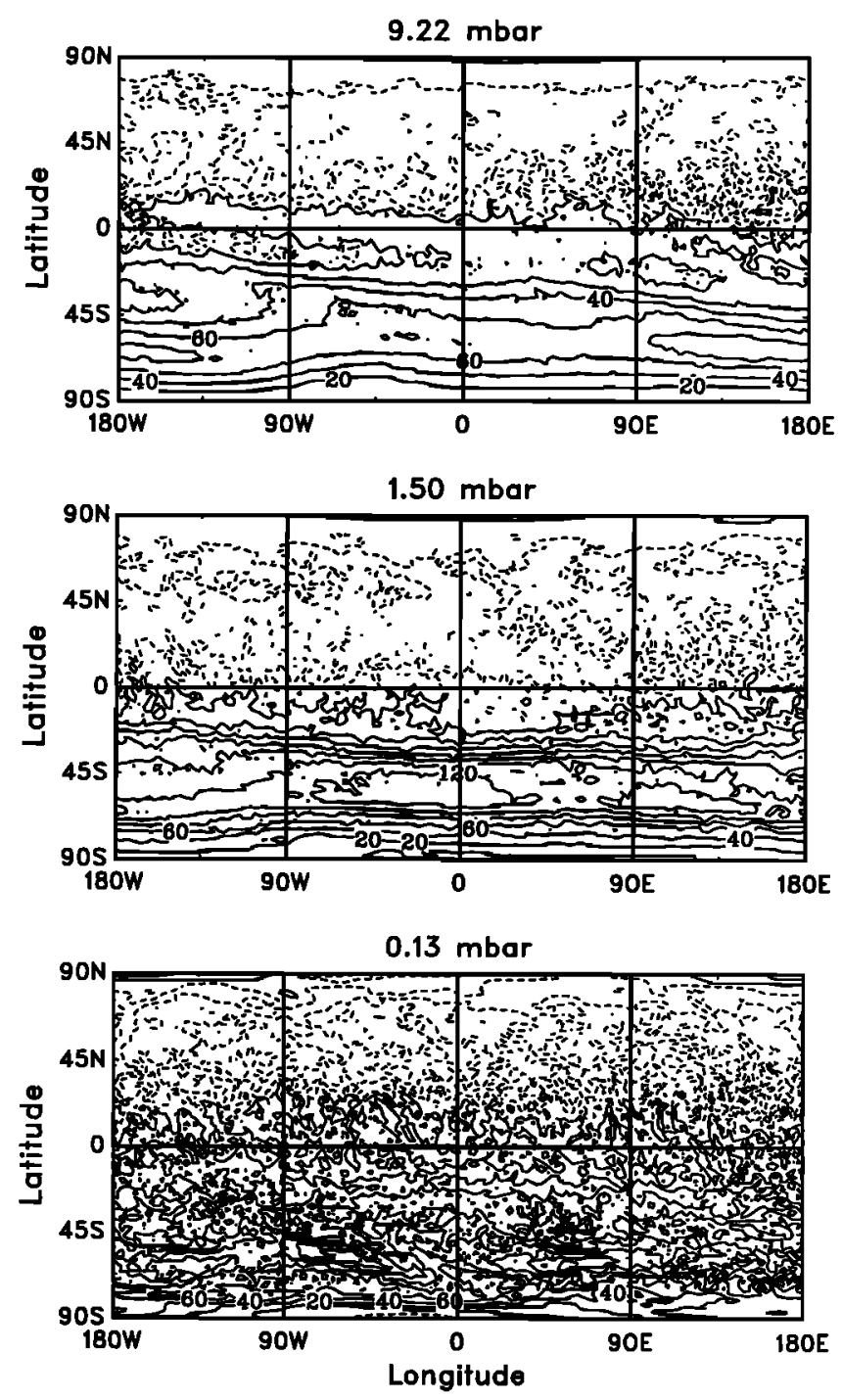

Figure 10. Zonal wind field on three different SKYHI (N90) model levels for a single snapshot in July: 9.22 mbar (top), 1.50 mbar (middle), and 0.13 mbar (bottom). Contour interval $=20 \mathrm{~m} / \mathrm{s}$ for positive-valued contours and $10 \mathrm{~m} / \mathrm{s}$ for negative-valued contours.

tent with the character of the KE spectrum, the field in Figure 10 shows an increasing small-scale structure with height. Similar behavior is seen in the other models, as well as in temperature and geopotential height fields. At $\sim 10 \mathrm{mbar}, u$ is dominated by a large-scale pattern in the winter (southern) hemisphere associated with the stratospheric polar vortex. Near the top of the stratosphere, at $\sim 1$ mbar there is a noticeable smallscale component, particularly strong in the tropics. The mesosphere is completely dominated by small-scale features with the exception of extreme polar regions.

It would be highly desirable to validate Figure 10 and the middle atmospheric spectra calculated as part of this study against suitable observations. Unfortunately, current data assimilation models extend no higher than the stratopause and the credible aspects of current assimilation products span at most the largest decade of horizontal scales in the stratosphere. An interesting future possibility involves comparing frequency spectra computed from model fields at fixed points in space to frequency spectra computed from lidar or radar data at the same points in the atmosphere.

Acknowledgments. This study was motivated by the participation of the authors in GRIPS, the GCM Reality Intercomparison Project for SPARC (Stratospheric Processes and Their Role in Climate). The authors thank Ted Shepherd and two anonymous reviewers for many useful insights that have been used to improve the paper.

\section{References}

Andrews, D. G., J. D. Mahlman, and R. W. Sinclair, Eliassen-Palm diagnostics of wave-mean flow interactions in the GFDL SKYHI general circulation model, J. Atmos. Sci., 40, 2768-2784, 1983.

Andrews, D. G., J. R. Holton, and C. B. Leovy, Middle Atmosphere Dynamics, 489 pp., Academic, San Diego, Calif., 1987.

Baer, F., An alternate scale representation of atmospheric energy spectra, J. Atmos. Sci., 29, 649-664, 1972.

Balsley, B. B., and D. A. Carter, The spectrum of atmospheric velocity fluctuations at $8 \mathrm{~km}$ and $86 \mathrm{~km}$, Geophys. Res. Lett., 9, 465-468, 1982.

Barat, J., Some characteristics of clear air turbulence in the middle atmosphere, J. Atmos. Sci., 39, 2553-2564, 1982.

Beagley, S. R., J. deGrandpré, J. N. Koshyk, N. A. McFarlane, and T. G. Shepherd, Radiative-dynamical climatology of the first-generation Canadian middle atmosphere model, Atmos. Ocean, 35, 293-331, 1997.

Boer, G. J., Homogeneous and isotropic turbulence on the sphere, J. Atmos. Sci., 40, 154-163, 1983.

Boer, G. J., and T. G. Shepherd, Large-scale twodimensional turbulence in the atmosphere, J. Atmos. Sci., 40, 164-184, 1983.

Boer, G. J., N. A. McFarlane, R. Laprise, J. D. Henderson, and J.-P. Blanchet, The Canadian Climate Centre spectral atmospheric general circulation model, Atmos. Ocean, 22, 397-429, 1984.

Boville, B. A., Wave-mean flow interactions in a general circulation model of the troposphere and stratosphere, $J$. Atmos. Sci., 43, 1711-1725, 1986.

Boville, B. A., Middle atmosphere version of CCM2 (MACCM2): Annual cycle and interannual variability, $J$. Geophys. Res., 100, 9017-9039, 1995.

Carter, D. A., and B. B. Balsley, The summer wind field between 80 and $93 \mathrm{~km}$ observed by the MST radar at Poker Flat, Alaska (65 $\left.{ }^{\circ} \mathrm{N}\right), J$. Atmos. Sci., 39, 2905-2915, 1982.

Charney, J. G., Geostrophic turbulence, J. Atmos. Sci., 28, 1087-1095, 1971.

Charney, J. G., and P. G. Drazin, Propagation of planetaryscale disturbances from the lower into the upper atmosphere, J. Geophys. Res., 66, 83-109, 1961.

Chen, T.-C., and A. Wiin-Nielsen, Non-linear cascades of atmospheric energy and enstrophy in a two-dimensional spectral index, Tellus, 30, 313-322, 1978.

Gao, X., J. W. Meriwether, V. B. Wickwar, T. D. Wilkerson, and $S$. Collins, Rayleigh lidar measurements of the temporal frequency and vertical wavenumber spectra in the mesosphere over the Rocky Mountain region, J. Geophys. Res., 103, 6405-6416, 1998.

Gregory, D., and P. R. Rowntree, A mass flux convection scheme with representation of cloud ensemble characteristics and stability dependent closure, Mon. Weather Rev., 118, 1483-1506, 1990. 
Hack, J. J., Parameterization of moist convection in the NCAR Community Climate Model CCM2, J. Geophys. Res., 99, 5551-5568, 1994.

Hamilton, $K$., What we can learn from general circulation models about the spectrum of middle atmospheric motions, in Coupling Processes in the Lower and Middle Atmosphere, edited by E.V. Thrane et al., pp. 161-174, Kluwer Acad., Norwell, Mass., 1993.

Hamilton, K., and J. D. Mahlman, General circulation model simulation of the semiannual oscillation of the tropical middle atmosphere, J. Atmos. Sci., 45, 3212-3235, 1988.

Hamilton, K., R. J. Wilson, J. D. Mahlman, and L. J. Umscheid, Climatology of the SKYHI tropospherestratosphere-mesosphere general circulation model, $J$. Atmos. Sci., 52, 5-43, 1995.

Hayashi, Y., D. G. Golder, J. D. Mahlman, and S. Miyahara, The effect of horizontal resolution on gravity waves simulated by the GFDL SKYHI general circulation model, Pure Appl. Geophys., 130, 421-443, 1989.

Hines, C. O., Internal atmospheric gravity waves at ionospheric heights, Can. J. Phys., 38, 1441-1481, 1960.

Holloway, J. S., and S. Manabe, Simulation of climate by a global general circulation model, I, Hydrological cycle and heat balance, Mon. Weather Rev., 99, 335-370, 1971.

Koshyk, J. N., and G. J. Boer, Parameterization of dynamical subgrid-scale processes in a spectral GCM, J. Atmos. Sci., 52, 965-976, 1995.

Koshyk, J. N., K. Hamilton, and J. D. Mahlman, Simulation of the $k^{-5 / 3}$ mesoscale spectral regime in the GFDL SKYHI general circulation model. Geophys. Res. Lett., 26, 843-846, 1999.

Kraichnan, R., Inertial ranges in two-dimensional turbulence, Phys. Fluids, 10, 1417-1423, 1967.

Lambert, S. J., A global available potential energy-kinetic energy budget in terms of the two-dimensional wavenumber for the FGGE year, Atmos. Ocean, 22, 265-282, 1984.

Laursen, L., and E. Eliasen, On the effects of the damping mechanisms in an atmospheric general circulation model, Tellus, Ser. A, 41, 385-400, 1989.

Leith, C. E., Atmospheric predictability and twodimensional turbulence, J. Atmos. Sci., 28, 145-161, 1971.

Manzini, E., and K. Hamilton, Middle atmosphere traveling waves forced by latent and convective heating, J. Atmos. Sci., 50, 2180-2200, 1993.

Manzini, E., N. A. McFarlane, and C. McLandress, Impact of the Doppler spread parameterization on the simulation of the middle atmosphere circulation using the MA/ECHAM4 general circulation model, J. Geophys. Res., 102, 25,751-25,762, 1997.

McIntyre, M. E., and T. N. Palmer, Breaking planetary waves in the stratosphere, Nature, 305, 593-600, 1983.

Miyahara, S., Y. Hayashi, and J. D. Mahlman, Interaction between gravity waves and planetary-scale flow simulated by the GFDL SKYHI general circulation model, J. Atmos. Sci., 43, 1844-1861, 1986.

Moorth, A., and M. J. Suarez, Relaxed Arakawa-Schubert: A parameterization of moist convection for general circulation models, Mon. Weather Rev., 120, 978-1002, 1992.

Nastrom, G. D., K. S. Gage, and W. H. Jasperson, Kinetic energy spectrum of large- and mesoscale atmospheric processes, Nature, 310, 36-38, 1984.

Nordeng, T. E., Extended versions of the convective parameterization scheme at ECMWF and their impact on the mean and transient activity of the model in the tropics, ECMWF Tech. Memo. 206, 41 pp., Reading, England, 1994.

Sato, T., and R. F. Woodman, Fine altitude resolution observations of stratospheric turbulent layers by the Arecibo $430 \mathrm{MHz}$ radar, J. Atmos. Sci., 39, 2546-2552, 1982.

Shibata, K., H. Yoshimura, M. Ohizumi, M. Hosaka, and M. Sugi, A simulation of the troposphere, stratosphere and mesosphere with the MRI/JMA98 GCM, Pap. Meteorol. Geophys., 50, 15-53, 1999.

Swinbank, R., and A. O'Neill, A stratosphere-troposphere data assimilation system, Mon. Weather Rev., , 122, 686$702,1994$.

Tang, C.-M., and S. A. Orszag, Two-dimensional turbulence on the surface of a sphere, J. Fluid Mech., 872, 305-318, 1978.

Tiedtke, M., A comprehensive mass flux scheme for cumulus parameterization in large-scale models, Mon. Weather Rev., 117, 1779-1800, 1989.

Vincent, R. A., and S. M. Ball, Mesospheric winds at lowand mid-latitudes in the southern hemisphere, J. Geophys. Res., 86, 9159-9169, 1981.

Zhang, G. J., and N. A. McFarlane, Sensitivity of climate simulations to the parameterization of cumulus convection in the CCC-GCM, Atmos. Ocean, 39, 407-446, 1995.

B. A. Boville, National Center for Atmospheric Research, Climate Modeling Section, P.O. Box 3000, Boulder, CO 80307. (boville@ncar.ucar.edu)

K. Hamilton, Geophysical Fluid Dynamics Laboratory/NOAA, Princeton University, Princeton, NJ 08542. (kph@gfdl.gov)

J. N. Koshyk, Department of Physics, University of Toronto, Toronto, Ontario M5S 1A7, Canada. (koshyk@mam.physics.utoronto.ca)

E. Manzini, Max Planck Institut für Meteorologie, Bundestrasse 55, 20146 Hamburg, Germany. (manzini@dkrz.de)

K. Shibata, Meteorological Research Institute, Climate Research Department, 1-1 Nagamine, Tsubuka, Ibaraki 3050052, Japan. (kshibata@mri-jma.go.jp)

(Received December 4, 1998; revised July 14, 1999; accepted July 21, 1999.) 